\title{
El pluralismo y el control de la información en las televisiones generalistas españolas
}

\author{
Xosé Soengas Pérez \\ Universidad de Santiago de Compostela \\ jose.soengas@usc.es
}

Recibido: 5 de diciembre de 2014

Aceptado: 8 de mayo de 2015

\begin{abstract}
Resumen
El objetivo de esta investigación es analizar y comparar los informativos de TVE, de Antena 3, de Cuatro, de Telecinco y de La Sexta, emitidos en 2008 y en 2013, para conocer el grado de pluralismo que ofrecen las televisiones generalistas españolas. Los datos demuestran que la existencia de varias emisoras no garantiza una oferta informativa plural y diversa. Los continuos cambios de criterio con respecto al tratamiento periodístico del mismo tema confirman que la línea editorial no está basada exclusivamente en el rigor. Los enfoques reflejan que existe un control de la información permanente y que los intereses políticos y económicos de las cadenas influyen en las versiones de las noticias e impiden que los ciudadanos conozcan muchos aspectos de la realidad.
\end{abstract}

Palabras clave: pluralismo, control información, televisión.

\section{Pluralism and information control in the Spanish general-interest channels}

\begin{abstract}
The aim of this research is to analyse and compare the newscast of the Spanish channels TVE, Antena 3,Telecinco and La Sexta aired in 2008 and 2013 in order to acknowledge the pluralism level in these generalist channels. Data shows that the presence of multiple TV channels does not guarantee a plural and diverse informative offer to the viewer. The continuous change in criteria regarding the journalistic treatment of a common topic suggest that the editorial line is not based in accuracy. These approaches reflect the existence of a permanent information control and that political and economic interests influence the versions of the news, blocking some aspects of reality to the citizens.
\end{abstract}

Keywords: pluralism, information control, television.

\section{Referencia normalizada}

SOENGAS PÉREZ, Xosé (2015): "El pluralismo y el control de la información en las televisiones generalistas españolas". Estudios sobre el Mensaje Periodístico. Especial noviembre "Periodismo e información de calidad", págs.: 199-207. Madrid, Servicio de Publicaciones de la Universidad Complutense.

Sumario: 1. Introducción y marco teórico; 1.1. La oferta informativa actual; 1.2. La información, un bien público; 1.3. Las dependencias de las televisiones. 2. Objeto de estudio. 3. Objetivos e hipótesis. 4. Metodología. 5. Análisis de los datos y resultados; 5.1. El pluralismo formal; 5.2. El pluralismo real; 5.3. El control de la información. 6. Conclusiones. 7. Referencias bibliográficas.

\section{Introducción y marco teórico}

\subsection{La oferta informativa actual}

El número de canales de televisión generalista que hay en España en estos momentos es suficiente para ofrecer y mantener una oferta informativa de calidad (Soler Campillo, 2005: web), pero la existencia de muchas televisiones no garantiza el pluralismo ni la diversidad porque pueden existir intereses comunes que generan un consenso sobre el tratamiento de determinados temas. Además, la pertenencia de varias emisoras al mismo grupo mediático favorece el control de los contenidos y aumenta la probabilidad de que predominen discursos adaptados a intereses concretos. Este aspecto 
es determinante porque, para analizar con rigor los contenidos de los informativos, es necesario conocer la propiedad de los medios y saber cuáles son los intereses políticos, económicos e ideológicos de los dueños de las cadenas (Reig, 2011), que son los factores determinantes de la línea editorial que establece cada emisora.

La información es un valor que trasciende el ámbito periodístico porque repercute en el terreno político, económico y social (Castillo Esparcia, 2012: web), por eso su rentabilidad se mide también desde estos tres parámetros, aunque los medios públicos no deberían buscar la rentabilidad política, ni la económica porque ambas afectan al pluralismo, a la neutralidad y a la independencia de los redactores (Ortells Badenes, 2009: web). Los objetivos económicos de las televisiones privadas, combinados con los intereses políticos y con el control gubernamental de TVE (Pérez Ornia, 1989), influyen en la oferta informativa e imponen criterios que no son compatibles con la calidad y con el rigor periodístico (Gómez Mompart et al., 2013). TVE debe considerar la rentabilidad de la información desde una óptica de servicio público y no desde intereses políticos, económicos o ideológicos, y mantener una línea editorial basada exclusivamente en criterios profesionales y ajena a los cambios drásticos que se producen cada vez que toma posesión un Gobierno nuevo (Soler Campillo, 2005: web).

La estructura de TVE, de Antena 3, de Cuatro, de Telecinco y de La Sexta es operativa, pero su organigrama actual es vulnerable y permite que haya injerencias políticas que fomentan la politización de la información (Casero Ripollés, 2008: web). El control político y gubernamental de los medios (Pérez Ornia, 1989), unido a la dependencia económica, acentuada por la crisis en el sector de la comunicación, favorece las intromisiones en la independencia profesional y limita la libertad de acción de los redactores (Segado Boj et al., 2013).

Las televisiones privadas se rigen por criterios comerciales porque su objetivo principal es obtener beneficios económicos, pero también buscan la rentabilidad política y ambos fines se retroalimentan. TVE, aunque es un medio público, funciona con el mismo esquema de las emisoras privadas, algo que contradice su esencia (Bustamante, 2013). En la mayoría de los casos hay intereses cruzados e interrelacionados, porque los vínculos entre la política, la economía y el periodismo son habituales (Casero Ripollés, 2008: web). Además, al predominar los criterios comerciales, las estrategias para conseguir una audiencia elevada influyen en la selección temática, en el enfoque de las noticias y en el tratamiento informativo de los hechos, y a veces los objetivos comerciales y los intereses políticos son incompatibles con los criterios informativos (García Avilés, 2007: web).

\subsection{La información, un bien público}

Al comparar las televisiones privadas con TVE somos conscientes de que los objetivos, la responsabilidad y la función que deben cumplir no es la misma desde el punto de vista de la programación general (De Mateo y Bergés, 2008). Pero consideramos que la información es un bien público y un derecho de los ciudadanos siempre, y cualquier medio, independientemente de su titularidad o de las características del soporte, en el ámbito informativo, tiene unas obligaciones y unas responsabilidades concretas frente a la sociedad que le obligan a cumplir unos requisitos de calidad, de pluralismo, 
de rigor, de imparcialidad y de neutralidad, para ofrecer un periodismo de calidad y con valores (Gómez Mompart et al., 2013). Y como la información es un bien público, la selección temática y el tratamiento de los contenidos deben estar basados en criterios de actualidad, responder al interés general y no regirse por imposiciones y por fórmulas que fomentan la espectacularización para conseguir audiencia (León, 2010). Los informativos deben reflejar el pluralismo social a través de la diversidad de opiniones y la variedad de temas y de fuentes (Humanes et al., 2013: web), con un tratamiento equitativo de todos los puntos de vista de la noticia, tanto en el aspecto formal como en el enfoque.

\subsection{Las dependencias de las televisiones}

La independencia de las televisiones es determinante para garantizar el pluralismo, evitar el control o el monopolio de la información e impedir injerencias de partidos políticos o de sectores interesados en limitar el ejercicio del periodismo y la libertad de información (Humanes et al., 2013: web). Las ayudas económicas que conceden las administraciones a los medios siempre conllevan ataduras. En el contexto actual, la crisis económica ha afectado de una forma especial al sector de la prensa, debido a la importante disminución de los ingresos procedentes de la publicidad, y ha aumentado la precariedad laboral, la dependencia y la vulnerabilidad de los medios, algo que repercute en los contenidos y en las condiciones profesionales de los periodistas (Soengas et al., 2014: web). La estabilidad laboral es determinante para hacer frente a posibles presiones externas e internas. Para ejercer el periodismo en condiciones óptimas es necesario disponer de los recursos humanos y materiales adecuados para resolver las dificultades que puedan presentarse durante una cobertura informativa. Es importante evitar impedimentos que pongan en riesgo las garantías necesarias que deben existir durante el proceso de producción de la información. Para que exista un periodismo de calidad es necesario un modelo de televisión sostenible, sin ataduras políticas ni económicas, que garantice el pluralismo, el rigor de los contenidos y la independencia de los redactores, con una línea editorial que no esté supeditada a presiones circunstanciales (Soler Campillo, 2005: web).

\section{Objeto de estudio}

El objeto de estudio de esta investigación se centra en el análisis del tratamiento informativo que han tenido en TVE, en Antena 3, en Cuatro, en Telecinco y en La Sexta la crisis económica, el paro, la sanidad, la educación y las reivindicaciones autonómicas durante 2008 y 2013, dos años en los que gobernaron el PSOE y el PP, respectivamente.

Seleccionamos estos temas porque son transversales y de interés general, y tienen repercusiones en el ámbito político, económico y social, han tenido vigencia y han sido referencia informativa en los dos años señalados. Además, son cuestiones que han protagonizado la acción de Gobierno y las reacciones de la oposición, y sobre las que el PSOE y el PP mantienen posiciones distintas. También son contenidos representativos de los aspectos que configuran el discurso ideológico y definen las principales líneas de actuación de ambos partidos. Por eso son claves para analizar las 
características de la línea editorial de cada televisión y para comprobar si se mantienen siempre los mismos criterios informativos o si varían en alguna emisora cuando cambia el Gobierno, y son temas adecuados para conocer el grado de control y de pluralismo real que existe en cada televisión.

Trabajamos con una muestra que comprende los años 2008 y 2013, y de cada año seleccionamos 12 informativos mediante el método de la semana compuesta, de tal forma que quedan representados todos los meses del año y todos los días de la semana. Y de esos informativos sólo analizamos los contenidos que se corresponden con los temas señalados en el objeto de estudio.

\section{Objetivos e hipótesis}

El objetivo de esta investigación es conocer el grado de control de la información y el grado de pluralismo real que existe en TVE, en Antena 3, en Cuatro, en Telecinco y en La Sexta. Y también nos interesan las características de la línea editorial de cada emisora y comprobar si el cambio de Gobierno influye en el enfoque y en el tratamiento de los contenidos.

Partimos de las siguientes hipótesis:

La existencia de varias televisiones no garantiza el pluralismo ni la diversidad en la oferta informativa.

El control de la información es permanente, pero no afecta siempre igual a los mismos temas. Las injerencias externas influyen en los enfoques de las noticias y determinan el cambio de criterio según los intereses políticos y económicos de cada momento.

Las televisiones privadas tienen una línea editorial más estable que TVE, que cambia su política informativa cada vez que se renueva el Gobierno.

\section{Metodología}

Además de los criterios técnicos que se aplican para editar con coherencia una noticia en televisión, existen otros requisitos para garantizar que en el relato audiovisual tengan presencia todos los puntos de vista necesarios. Por eso, para conocer el grado de pluralismo formal (Humanes et al., 2013: web), nos interesa saber si en las noticias analizadas están incluidos, desde un parámetro cuantitativo, todos los elementos esenciales para que el acontecimiento conserve su identidad inicial y la versión sea correcta (González, 1989: web). Y para determinar el grado de pluralismo real tenemos en cuenta los aspectos formales y el análisis de contenido, los criterios cuantitativos y los cualitativos, y comparamos la correspondencia y el equilibrio entre ambos aspectos.

De acuerdo con los objetivos de esta investigación, realizamos un análisis de contenido, y también comparativo y longitudinal. Esta metodología mixta permite analizar conjuntamente todos los elementos que forman parte de las diferentes dimensiones del relato informativo, comparar y contrastar los datos, conocer el tratamiento de los temas seleccionados y ver si hubo cambios significativos en alguna televisión entre 2008 y 2013.

En una información existen aspectos que no se pueden valorar objetivamente desde un parámetro estrictamente cuantitativo, aquellos que forman parte de los márgenes 
interpretativos que posibilitan los múltiples enfoques que puede tener una noticia. Por eso es necesario un análisis cualitativo para conocer los detalles que generan las distintas versiones de un hecho. $Y$ esos matices son los que permiten identificar, definir y conocer las características de la línea editorial, comprobar si hubo cambios de criterio en la política informativa, y determinar de una forma específica el grado de control de la información que existe en cada televisión.

Al establecer unos criterios que permiten identificar los elementos esenciales de una noticia y comprobar si esa información reúne esos requisitos (González, 1989: web), podemos reconocer los posibles enfoques y múltiples puntos de vista legítimos y diferenciarlos de la censura y de la manipulación. Así es posible ver si existen carencias informativas en algún tema, valorar con rigor los contenidos y establecer unos parámetros que permitan calcular los índices de calidad y de objetividad (Gómez Mompart et al., 2013).

Al centrar el objeto de estudio en los mismos temas emitidos por televisiones con líneas editoriales distintas y en años diferentes, 2008 y 2013, el análisis comparativo y longitudinal permite comprobar si existen coincidencias o diferencias en el tratamiento informativo de esos contenidos, y también permite determinar si permanecen los mismos criterios durante las dos etapas analizadas, o si alguna de las cadenas ha modificado su política informativa. Así es posible valorar mejor la dimensión de cualquier cambio, sus particularidades y su alcance, tanto en el tratamiento formal como en el enfoque.

\section{Análisis de los datos y resultados}

\subsection{El pluralismo formal}

El análisis de las noticias seleccionadas demuestra que las televisiones objeto de estudio cumplen los requisitos formales en la mayoría de los casos, una constante que se mantiene en las muestras de 2008 y en las de 2013.

Según los criterios aplicados en esta investigación para determinar el grado de pluralismo formal (Humanes et al., 2013: web), el 97\% de las noticias de TVE analizadas en 2008 incluyen todos los puntos de vista necesarios, un porcentaje muy similar al registrado en Antena 3 (91\%), en Cuatro (95\%), en Telecinco (96\%) y en La Sexta (93\%).

Los datos de 2013 ratifican que los informativos mantienen un grado de pluralismo formal alto. En el 93\% de las noticias de TVE aparecen representados adecuadamente todos los puntos de vista. Y en las televisiones privadas los porcentajes son casi idénticos: (90\%) en Antena 3, (95\%) en Cuatro, (94\%) en Telecinco y (91\%) en La Sexta.

\subsection{El pluralismo real}

Uno de los objetivos de la investigación es conocer el grado de pluralismo real que existe en la oferta informativa que hacen las televisiones generalistas españolas en 2008 y en 2013. Por eso, después de analizar las características formales de las noticias, realizamos un análisis de contenido. Aquí valoramos el enfoque y el tratamiento informativo para determinar el grado de neutralidad y de pluralidad, y ver si en alguna televisión existen elementos novedosos que aportan otro punto de vista y configuran una oferta informativa diferenciada con respecto a las demás cadenas. 
Los resultados del análisis de contenido demuestran que el grado de pluralismo formal registrado en 2008 y en 2013 no se corresponde con el grado de neutralidad ni de pluralismo real localizado en los informativos de esos mismos años. Los detalles de los aspectos cualitativos, además de definir la línea editorial de la emisora, demuestran que existen enfoques que no se ajustan a un tratamiento objetivo de los hechos, de acuerdo con los parámetros utilizados como referencia en esta investigación (González, 1989: web).

Para determinar el grado de pluralismo real que existe en la oferta informativa de las televisiones generalistas españolas elaboramos unos indicadores que permiten comparar íntegramente las versiones que ofrecen de los temas seleccionados como objeto de estudio TVE, Antena 3, Cuatro, Telecinco y La Sexta. Así obtenemos el grado de coincidencia que se da entre las diferentes emisoras y, al mismo tiempo, los niveles de pluralismo real, que sirven como indicador para determinar el grado de control de la información.

La siguiente tabla incluye los resultados del análisis comparativo y longitudinal, y refleja el grado de coincidencia que existe entre los enfoques y los tratamientos informativos de cada televisión, con respecto a los mismos contenidos emitidos en las demás emisoras, en 2008 y en 2013.

Tabla 1: Grado de coincidencia entre los enfoques de las diferentes televisiones en 2008 y 2013. Fuente: Elaboración propia

\begin{tabular}{|c|c|r|r|r|r|}
\hline \multicolumn{6}{|c|}{ Grado de coincidencia entre los enfoques de las diferentes } \\
televisiones en 2008 \\
\hline Cadenas & TVE & A3 & Cuatro & \multicolumn{1}{c|}{ T5 } & La Sexta \\
\hline TVE & $100 \%$ & $35 \%$ & $78 \%$ & $84 \%$ & $81 \%$ \\
\hline A3 & $35 \%$ & $100 \%$ & $38 \%$ & $27 \%$ & $30 \%$ \\
\hline Cuatro & $78 \%$ & $38 \%$ & $100 \%$ & $88 \%$ & $79 \%$ \\
\hline T5 & $84 \%$ & $27 \%$ & $88 \%$ & $100 \%$ & $90 \%$ \\
\hline La Sexta & $81 \%$ & $30 \%$ & $79 \%$ & $90 \%$ & $100 \%$ \\
\hline \multicolumn{6}{|c|}{ Grado de coincidencia entre los enfoques de las diferentes } \\
\hline Cadenas & TVE & $\mathbf{A 3}$ & Cuatro & T5 & La Sexta \\
\hline TVE & $100 \%$ & $89 \%$ & $37 \%$ & $31 \%$ & $33 \%$ \\
\hline A3 & $89 \%$ & $100 \%$ & $42 \%$ & $38 \%$ & $29 \%$ \\
\hline Cuatro & $37 \%$ & $42 \%$ & $100 \%$ & $85 \%$ & $83 \%$ \\
\hline T5 & $31 \%$ & $38 \%$ & $85 \%$ & $100 \%$ & $87 \%$ \\
\hline La Sexta & $33 \%$ & $29 \%$ & $83 \%$ & $87 \%$ & $100 \%$ \\
\hline
\end{tabular}

\subsection{El control de la información}

En 2008 la situación socioeconómica de España era muy diferente a la de 2013 y esta circunstancia se refleja en el discurso informativo, pero en ambos casos se percibe una alineación de las televisiones con el Gobierno o con la oposición, tal como se comprueba al comparar los enfoques de las dos etapas. El discurso dominante que mantienen las emisoras analizadas en 2008 contradice los criterios que aplican en 2013 sobre los mismos temas, y el caso más evidente es el de TVE.

El análisis de contenido refleja que en 2008, año en el que gobernaba el PSOE, hay un alto grado de coincidencia entre el enfoque y el tratamiento informativo que hacen 
TVE, Cuatro, Telecinco y La Sexta de los temas seleccionados como objeto de estudio con los criterios, las tesis y las acciones del Gobierno sobre esos mismos contenidos, lo cual demuestra que existe una alineación permanente entre estas televisiones y el Ejecutivo. En cambio, el enfoque y el tratamiento informativo que hace Antena 3 sobre las mismas cuestiones es muy diferente y se caracteriza por un discurso crítico con las propuestas institucionales.

En 2008 en TVE el 83\% de las noticias analizadas tienen un enfoque que coincide con los criterios del Gobierno, un porcentaje muy similar al que se registra en Cuatro (78\%), en Telecinco (86\%) y en La Sexta (81\%). En cambio, en Antena 3 las coincidencias entre las tesis del Ejecutivo y los enfoques de la cadena sólo representan el $11 \%$, mientras que las versiones críticas alcanzan el $89 \%$.

En 2013 gobierna el PP y aquí los criterios informativos cambian radicalmente. En esta etapa las coincidencias ya no se dan entre las mismas cadenas que en 2008. El discurso de TVE ahora coincide con el de Antena 3 y presenta discrepancias con los planteamientos de Cuatro, de Telecinco y de La Sexta, que son críticos con las acciones del Gobierno. En esta etapa se aprecia una mayor politización de TVE porque aumenta el porcentaje de informaciones cuyo enfoque favorece al Gobierno (92\%), algo que se traduce en un mayor control de los contenidos. En Antena 3 también destacan los tratamientos próximos al Ejecutivo (81\%), pero en las demás emisoras son minoría los enfoques que coinciden con las tesis del Gobierno: Cuatro (21\%), Telecinco $(20 \%)$ y La Sexta (17\%).

\section{Conclusiones}

Los resultados confirman las hipótesis planteadas en la investigación.

El grado de pluralismo formal que se registra en los informativos de TVE, de Antena 3, de Cuatro, de Telecinco y de La Sexta es alto, en 2008 y en 2013, pero no se corresponde con el grado de pluralismo real, ni con los índices de objetividad que se aprecian en el análisis de contenido. El grado de control informativo que se advierte en las emisoras analizadas es incompatible con la responsabilidad que les corresponde a las televisiones y con los estándares de calidad exigibles a un medio de comunicación independiente.

El mapa televisivo actual favorece la existencia de dos discursos informativos previsibles, alineados, en la mayoría de los casos, de forma permanente con las tesis y con las propuestas del PSOE y del PP, especialmente en las noticias de contenido político y en las informaciones que afectan a la acción de Gobierno. Los resultados demuestran que las líneas editoriales de las televisiones están marcadas por criterios ideológicos y que la existencia de varias emisoras no garantiza una oferta informativa plural y diversa.

Los enfoques y los tratamientos informativos reflejan que existe un control de la información permanente y que los intereses políticos y económicos de las cadenas influyen en las versiones de las noticias.

Los cambios drásticos en la línea editorial de TVE, si comparamos los criterios vigentes en 2008 con los aplicados por la dirección de informativos en 2013, demuestran que existe un control exhaustivo de la televisión pública por parte del Gobierno. 


\section{Referencias bibliográficas}

BUSTAMANTE, Enrique (2013): Historia de la Radio y la Televisión en España. Una asignatura pendiente de la democracia. Barcelona, Gedisa.

CASERO RIPOLLÉS, Andreu (2008): "Modelos de relación entre periodistas y políticos: la perspectiva de la negociación constante", en Estudios sobre el Mensaje Periodístico: http://revistas.ucm.es/index.php/ESMP/article/view/ESMP080811 0111A [Consulta: 8 de mayo de 2014]. Madrid, Servicio de Publicaciones de la Universidad Complutense.

CASTILLO ESPARCIA, Antonio (2012): "Los medios de comunicación como actores sociales y políticos. Poder, medios de comunicación y sociedad", en Razón y Palabra: http://www.razonypalabra.org.mx/N/N75/monotematico_75/12_Casti1lo_M75.pdf [Consulta: 25 de mayo de 2014]

DE MATEO, Rosario y BERGÉS, Laura (2008): Los retos de las televisiones públicas. Financiación, servicio público y libre mercado. Sevilla, Comunicación Social.

GARCÍA AVILÉS, José Alberto (2007): "El infoentretenimiento en los informativos líderes de audiencia en la Unión Europea”, en Anàlisi: http://ddd.uab.cat/pub/analisi/02112175n35p47.pdf [Consulta: 23 de abril de 2014]

GÓMEZ MOMPART, Josep Lluís; GUTIÉRREZ LOZANO, Juan; y PALAU SAMPIO, Dolors (eds., 2013): La calidad periodística. Teorías, investigaciones y sugerencias profesionales. Valencia, Servei de Publicacions Universitat de Valencia.

GONZÁLEZ, Norberto (1989): "Hechos y valores en la narración periodística informativa", en Comunicación y Sociedad: http:/www.unav.es/fcom/comunicacionysociedad/es/articulo.php?art_id=295 [Consulta: 6 de mayo de 2014]

HUMANES, María Luisa; MONTERO SÁNCHEZ, María Dolores; MOLINA DE DIOS, Ramón; y LÓPEZ-BERINI, Alfredo (2013): "Pluralismo y paralelismo político en la información televisiva en España". Revista Latina de Comunicación Social: $\mathrm{http}: / /$ plataformarevistascomunicacion.org/2013/10/pluralismo-y-paralelismopolitico-en-la-informacion-televisiva-en-espana/ [Consulta: 29 de mayo de 2014]

LEÓN, Bienvenido (coord., 2010): Informativos para la televisión del espectáculo. Sevilla y Zamora, Comunicación Social.

ORTELLS BADENES, Sara (2009): "La mercantilización de la información: la nueva era informativa en televisión", en Revista Latina de Comunicación Social: http://www.revistalatinacs.org/09/art/28_827_46_ULEPICC_07/Sara_Ortells.html [Consulta: 17 de abril de 2014]

PÉREZ ORNIA, José Ramón (1989): Peculiaridades de una televisión gubernamental. Barcelona, Ariel.

REIG, Ramón (2011): Los dueños del periodismo. Claves de la estructura mediática mundial y de España. Barcelona, Gedisa.

SEGADO BOJ, Francisco; FERNÁNDEZ GÓMEZ, Erika; GIL GASCÓN, Fátima; y MARTÍN QUEVEDO, Juan (2013): La crisis económica en los informativos de televisión. Logroño, Universidad Internacional de La Rioja. 
SOENGAS PÉREZ, Xosé; RODRÍGUEZ VÁZQUEZ, Ana Isabel; y ABUÍN VENCES, Natalia (2014): "La situación profesional de los periodistas españoles: las repercusiones de la crisis en los medios", en Revista Latina de Comunicación Social: http://www.revistalatinacs.org/069/paper/1003_USC/RLCS_paper1003.pdf [Consulta: 10 de mayo de 2014]

SOLER CAMPILLO, María (2005). "El informe del 'Comité de sabios': bases para una televisión de calidad", en Comunicar: http://www.revistacomunicar.com /index.php? contenido $=$ detalles\&numero $=25 \&$ articulo $=25-2005-104$ [Consulta: 21 de abril de 2014] 